\title{
Using Magnetic Nanoparticles to Eliminate Oscillations in Saccharomyces cerevisiae Fermentation Processes
}

\author{
Lakshmi N. Sridhar \\ Chemical Engineering Department, University of Puerto Rico, Mayaguez, Puerto Rico \\ Email: lakshmin.sridhar@upr.edu
}

Received June 4, 2012; revised July 11, 2012; accepted July 23, 2012

\begin{abstract}
This article provides computational evidence to show that functionalized magnetic nanoparticles can eliminate the wasteful oscillatory behavior in fermentation processes involving Saccharomyces cerevisiae. There has been a considerable amount of work demonstrating the existence of oscillations in fermentation processes. Recently reference [1] computationally demonstrated very simple strategies to eliminate the oscillations in the fermentation process. In the case of the of the Saccharomyces cerevisiae fermentation process it was shown that the addition of a little bit of oxygen would be successful in eliminating the oscillation causing Hopf bifurcations. The work of [2,3] demonstrated that oxygen mass transfer could be enhanced by using functionalized magnetic nanoparticles. The aim of this work is to incorporate the model used by [3] regarding the enhancement of oxygen mass transfer in the cybernetic Jones Kompala model [4] describing the dynamics of the Saccharomnyces cerevisiae fermentation process and demonstrate that using the functionalized magnetic nanoparticles can by altering the mass transfer coefficient actually succeed in eliminating the oscillatory behavior that plagues the Saccharomyces cerevisiae fermentation process. This occurs because the oscillation causing Hopf bifurcations are sensitive to the amount of input oxygen and increasing the oxygen mass transfer coefficient causes the disappearance of the Hopf bifurcation points.
\end{abstract}

Keywords: Nanoparticles; Fermentation; Oscillations

\section{Introduction}

Production of ethanol from a variety of biomass feedstocks, solid and liquid waste resources is a national priority for a variety of reasons but most importantly for energy independence and sustainability. The biggest hurdle in the commercialization of any of the processes for making ethanol is the cost of production. Hence, it is important to develop strategies to produce clean ethanol with the least amount of expense avoiding all unnecessary expenses and wastage during ethanol production.

Continuous fermentation processes used for ethanol production has been known to exhibit oscillatory behavior and this has been confirmed both theoretically and experimentally. Fermentation processes involving both Saccharomyces cerevisiae and Zymomonas mobilis have been shown to exhibit oscillatory behavior [5-11]. The oscillatory behavior was linked to the combination of substrate excess and product inhibitions by reference [8] while reference [10] conclude that the oscillations take place at high values of feed substrate concentrations.

References $[9,10,12,13]$ have demonstrated the existence of oscillatory behavior in continuous fermentations of Saccharomyces cerevisiae. While there have been a lot of modeling work regarding fermentation processes, the most sophisticated model available is the Jones Kompala model [4]. This model has demonstrated the observed oscillatory behavior [14,15]. Reference [4] discusses the effect of oxygen mass transfer on the Saccharomyces cerevisiae fermentation in a section in their paper titled "Effect of oxygen mass transfer on yeast oscillations".

The oscillatory behavior that has been demonstrated numerically and experimentally by several workers adversely affects the fermentation process and the ethanol production. Hence one must develop strategies to avoid or eliminate the oscillations. Reference [1] recently computationally demonstrated some techniques for eliminating oscillations in fermentation processes involving both Zymomonas mobilis and Saccharomyces cerevisiae. Specifically, it was shown that in the case of the Saccharomyces cerevisiae fermentation that a small increase in the input oxygen concentration would eliminate the oscillation causing Hopf bifurcation. This increase in oxygen mass transfer can also be observed if the mass transfer coefficient for the oxygen mass transfer can be enhanced. Recently, [2,3] investigated the use of functionalized magnetic nanoparticles with flourinated polymer coating to enhance oxygen mass transfer in bioreactors. The 
flourianted polymer coating actually aids in enhancing the mass transfer. The question that this paper addresses is "Can the use of functionalized magnetic nanopartices, by increasing the mass transfer of oxygen to the Saccharomyces cerevisiae fermentation process also eliminate the oscillatory behavior that occurs in these processes?" The oxygenation which is usually achieved by sparging air into the fermentation unit can be enhanced by 1) adding extra oxygen directly to the input stream and 2) increasing the mass transfer coefficient that pertains to the oxygen mass transfer. Reference [1] computationally demonstrated that the first strategy was effective in eliminating the oscillatory behavior in the Saccharomyces cerevisiae fermentation. This paper addresses the second issue.

While oscillatory behavior can cause wastage and affect the ethanol production adversely the constant addition of oxygen also can be expensive and if this expense can also be avoided it could be instrumental in reducing the cost of ethanol production.

The paper is organized as follows. First, the cybernetic Jones Kompala model [4] or the Saccharomyces cerevisiae fermentation is discussed. Then the Olle co-relation [3] for the increase in the mass transfer coefficient is described and incorporated into the cybernetic model. The reported cases for the oscillation causing Hopf bifurcations are presented and it is shown that when the mass transfer coefficient is enhanced by the use of the ferromagnetic nanoparticles, the Hopf bifurcations disappear in these cases.

\section{Saccharomyces cerevisiae Model}

Reference [4] have developed a detailed cybernetic model to represent the Saccharomyces cerevisiae fermentation process. Along three available pathways $r_{i}$, Glucose fermentation, ethanol oxidation and glucose oxidation, the cybernetic variables $u_{i}$ and $v_{i}$ represent the optimal strategies for enzyme synthesis and activity. The variables $u_{i}$ and $v_{i}$ are given by the equations

$$
\begin{gathered}
u_{i}=\frac{r_{i}}{\sum_{j} r_{j}} \\
v_{i}=\frac{r_{i}}{\max _{j} r_{j}}
\end{gathered}
$$

while the expressions for the pathways $r_{i}$ are given by

$$
\begin{gathered}
r_{1}=\mu_{1} e_{1} \frac{G}{K_{1}+G} \\
r_{2}=\mu_{2} e_{2}\left(\frac{E}{K_{2}+E}\right)\left(\frac{O}{K_{O_{2}}+O}\right)
\end{gathered}
$$

$$
r_{3}=\mu_{3} e_{3}\left(\frac{G}{K_{3}+G}\right)\left(\frac{O}{K_{O_{3}}+O}\right)
$$

with these growth rate equations, the balance equations [4] are given by

$$
\begin{gathered}
\frac{\mathrm{d} X}{\mathrm{~d} t}=X \sum_{i} r_{i} v_{i}-D X \\
\frac{\mathrm{d} G}{\mathrm{~d} t}=\left(G_{0}-G\right) D-\left(\frac{r_{1} v_{1}}{Y_{1}}-\frac{r_{2} v_{2}}{Y_{2}}\right) X-\phi_{4}\left(C \frac{\mathrm{d} X}{\mathrm{~d} t}+X \frac{\mathrm{d} c}{\mathrm{~d} t}\right) \\
\frac{\mathrm{d} E}{\mathrm{~d} t}=-D E+\left(\phi_{1} \frac{r_{1} v_{1}}{Y_{1}}-\frac{r_{2} v_{2}}{Y_{2}}\right) X \\
\left.\frac{\mathrm{d} e_{i}}{\mathrm{~d} t}=\alpha u_{i} \frac{S_{i}}{K_{i}+S_{i}}-\left(\sum_{j} r_{j} v_{j}+\beta\right) e_{i}+\alpha^{*}-O\right)-\left(\phi_{2} \frac{r_{2} v_{2}}{Y_{2}}+\phi_{3} \frac{r_{3} v_{3}}{Y_{3}}\right) X \\
\frac{\mathrm{d} C}{\mathrm{~d} t}=\gamma_{3} r_{3} v_{3}-\left(\gamma_{1} r_{1} v_{1}+\gamma_{2} r_{2} v_{2}\right) C-\sum_{i}\left(r_{i} v_{i}\right) C
\end{gathered}
$$

$G, E$ and $O$ represent the concentrations of glucose, ethanol and dissolved oxygen. $\mu_{i}$ represents the modified growth rate constant. $K_{i}$ and $K_{O i}$ represent the saturation constants for the carbon substrate and the dissolved oxygen for each metabolic pathway. $G_{0}$ represents the inlet glucose feed concentration, $X$ is the cell mass concentration and $k_{L} a$ represents the dissolved oxygen mass transfer coefficient. $Y$ is the yield coefficient, while $\alpha$ and $\beta$ represent the enzyme synthesis and decay rate constants. The stoichiometric coefficients for the intercellular storage carbohydrate synthesis and consumption are given by $\phi_{i}$ and $\gamma_{i}$. Table 1 gives the base values of the variables and constants used. This model demonstrates the existence of the Hopf bifurcations that cause the occurrence of the oscillatory behavior.

\section{Enhancement of Mass Transfer Coefficient Because of Functionalized Magnetic Nanoparticles}

References [2] and [3] has observed oxygen transfer enhancement in the presence of colloidal dispersions of magnetized nanoparticles coated with oleic acid and a polymerizable surfactant. In this work, fermentations in the presence of nanoparticles were conducted and it was demonstrated that the Oleic acid coated nanoparticles do enhance oxygen transfer rates and an empirical co-relation for the enhanced mass transfer coefficient in the presence of the nanopartiocles is presented as 


$$
K_{L, N P}=K_{L}(1+C \phi)
$$

$K_{L, N P}$ represents the enhanced mass transfer coefficient when the nanoparticles are added while $\phi$ represents the mass fraction of the nanoparticles. $C$ is an adjustable parameter which has been determined as 51.4. This linear relationship holds for mass fractions of nanoparticles up to $\phi \sim 0.01$. The value of $C$ was determined from experimental data and Equation (12) is an empirical one. We will use this relationship to investigate whether by adding the nanoparticles the oscillation causing Hopf bifurcations will disappear when the enhanced value of the mass transfer is used. The incorporation of the enhanced mass transfer coefficient will result in Equation (9) to be modified as

$$
\frac{\mathrm{d} O}{\mathrm{~d} t}=k_{L} a(1+C \phi)\left(O^{*}-O\right)-\left(\phi_{2} \frac{r_{2} v_{2}}{Y_{2}}+\phi_{3} \frac{r_{3} v_{3}}{Y_{3}}\right) X
$$

\section{Numerical Technique Used to Locate the Singularities}

The program CL_MATCONT $[16,17]$ was used to locate singularities in the set of ODE that constitute the Saccharomyces cerevisiae fermentation model (Equation set 6-11 and equation set $6,7,8,13,10,11)$. For the resulting equilibrium curve $f(u, \alpha)=0$, where $\alpha$ is the continuation parameter, and $u$ the remaining variables, the defining function is $F(x)=f(u, \alpha)=0$ where $x=(u, \alpha) \in R^{n+1}$ and $v$ is the tangent vector at $x$. Three test functions will be defined as

$$
\begin{aligned}
& \phi_{1}=\operatorname{det}\left(\begin{array}{c}
F_{x} \\
v^{T}
\end{array}\right) \\
& \phi_{2}=\operatorname{det}\left(2 f_{u}(u, \alpha)^{*} I_{n}\right) \\
& \phi_{3}(u, \alpha)=v_{n+1}
\end{aligned}
$$

For the existence of a branch point, $\phi_{1}=0$, while $\phi_{2}$ and $\phi_{3}$ are zero for a Hopf bifurcation point and a limit point. ${ }^{*}$ indicates the bialternate product.

\section{Results: Elimination of the Hopf Bifurcation Points in Saccharomyces cerevisiae Fermentation}

In this section, examples of Saccharomyces cerevisiae fermentation problems where the Hopf bifurcation points disappear when the mass transfer coefficients are enhanced because of the nanoparticles are presented. In all the cases $D$ is the bifurcation parameter. The same problems were investigated by [1] and the oscillation causing Hopf bifurcation points were eliminated there by increasing the input oxygen. The value of $O^{*}$ in all the cases was 7.5 which is the concentration of the oxygen in air. In all the cases studied it was seen that the addition of a small amount of the ferromagnetic nanoparticles results in the disappearance of the Hopf bifurcation.

Table 1 gives the base values for the parameters in the Jones Kompala model [4] and Table 2 provides the additional problem specifications and contains the mass fraction of the ferromagnetic nanoparticles required to eliminate the oscillation causing Hopf bifurcations. Table 3 provides the concentration values of the Hopf bifurcations before the addition of the ferromagnetic nanoparticles.

\section{Case 1 (Figures 1 and 2)}

The first Saccharomyces cerevisiae problem we study is the problem discussed in [15] In this case the value of $k_{L} a$ is 150 and the $G_{0}$ value is 10 . When the $O^{*}$ value is $7.5 \mathrm{mg} / \mathrm{l}$ there are 2 Hopf points, 5 neutral saddles and one limit point. This is indicated in Figure 1. When the mass fraction of the added ferromagnetic nanoparticles is 0.006 the Hopf bifurcations disappear. This is shown in Figure 2. Here 3 neutral saddles and a limit point remain. Neutral saddles are not bifurcation points and do not cause oscillations.

Table 1. Base set of parameters used for the Saccharomyces cerevisiae fermentation problem ([4]).

\begin{tabular}{cc}
\hline Parameter & Value \\
\hline $\mathrm{G}_{0}$ & $10 \mathrm{gm} / \mathrm{l}$ \\
$Y_{1}, Y_{2}, Y_{3}$ & $(0.16,0.75,0.6) \mathrm{gg}^{-1}$ \\
$\phi_{1}, \phi_{2}, \phi_{3}, \phi_{4}$ & $0.403,2,1,0.95$ \\
$O^{*}$ & $0.3 \mathrm{mg} / \mathrm{l}$ \\
$\alpha$ & 0.03 \\
$\alpha^{*}$ & 0.7 \\
$\beta$ & $0.05,0.01,0.001$ \\
$K_{1}, K_{2}, K_{3}$ & $0.01 \mathrm{mg} / \mathrm{l}$ \\
$K_{O_{2}}$ & $2.2 \mathrm{mg} / \mathrm{l}$ \\
$K_{O_{3}}$ & $10,10,0.8$ \\
$\gamma_{i}(i=1,2,3)$ & $0.44,0.19,0.36$ \\
$\mu_{i, \max }(i=1,2,3)$ & \\
\hline
\end{tabular}

Table 2. Problem specifications.

\begin{tabular}{cccc}
\hline Problem number & $G_{0}$ & $\begin{array}{c}\text { Original } \\
K_{L} a \\
\text { value }\end{array}$ & $\begin{array}{c}\phi \\
\text { required to eliminate } \\
\text { Hopf bifurcation }\end{array}$ \\
\hline 1 & 10 & 150 & 0.006 \\
2 & 11.5 & 225 & 0.005 \\
3 & 8.75 & 225 & 0.0065 \\
\hline
\end{tabular}


Table 3. Concentration values at the Hopf bifurcation points before ferromagnetic nanoparticles are added.

\begin{tabular}{|c|c|c|}
\hline Problem & $\begin{array}{l}\text { No. of Hopf points before } \\
\text { nanopartices addition }\end{array}$ & Hopf bifurcation co-ordinates $\left(X, C, G, E, O, e_{1}, e_{2}, e_{3}, D\right)$ \\
\hline 1 & 2 & 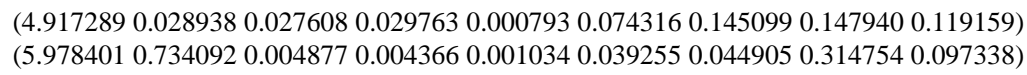 \\
\hline 2 & 1 & (6.873118 0.7322060 .0064750 .0060610 .0013370 .0386200 .0454100 .3169610 .121043$)$ \\
\hline 3 & 1 & (4.706247 0.1487030 .0386630 .1244650 .0019400 .0659380 .1207930 .2155180 .159153 ) \\
\hline
\end{tabular}

\section{Case 2 (Figures 3 and 4)}

In the second Saccharomyces cerevisiae example [14], for a $k_{L} a$ value of 225 , a $G_{0}$ value of 11.5 , and an $O^{*}$ value is $7.5 \mathrm{mg} / \mathrm{l}$, we get a Hopf bifurcation point, two neutral saddles and a limit point. This is shown in Figure 3. When the mass fraction of the ferromagnetic nanoparticles is 0.005 , the Hopf bifurcation disappears. The new curve is shown in Figure $\mathbf{4}$ where two neutral saddles and a limit point remain.

\section{Case 3 (Figures 5 and 6 )}

In the third Saccharomyces cerevisiae example, for a $k_{L} a$ value of 225 , a $G_{0}$ value of 8.75 , and an $O^{*}$ value is $7.5 \mathrm{mg} / \mathrm{l}$, we get one Hopf bifurcation point, four neutral saddles and a limit point. This is shown in Figure 3. When the mass fraction of the ferromagnetic nanoparticles is 0.0065 , the Hopf bifurcation disappears, as shown in Figure 6 and only the limit point remains.

\section{Discussion of Results}

The Jones Kompala model [4] is a powerful model hat is used to model the growth dynamics of Saccharomyces cerevisiae and predicts not only the oscillatory behavior but also the observed variations in the oscillations over a wide rang of dilution rates. Reference [18] experimentally studied the effect of oxygen mass transfer on oscillatory behavior in fermentation processes. In the cybernetic model of Jones and Kompala [4] the oxygen mass transfer is in the term $k_{L} a\left(O^{*}-O\right)$ and therefore enhancing the mass transfer coefficient would therefore have the same effect as increasing the input oxygen. While one of the most novel ways to increase the mass transfer coefficient is to use ferromagnetic nanoparticles this paper computationally incorporates the effect of the mass transfer enhancement in the cybernetic model of Jones and Kompala [4]. In the three cases, discussed the mass fraction of the nanoparticles required was 0.006, 0.005 and 0.0065 . It is interesting to note that the usage of a very minute amount of nanoparticles is effective in eliminating the oscillations in the fermentation processes. Having to use such a minute amount of the nanoparticles

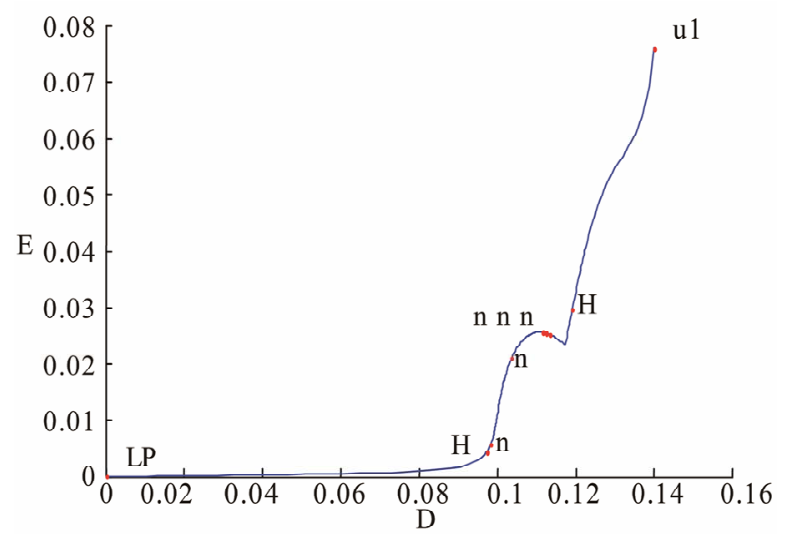

Figure 1. Hopf bifurcation for case 1 when no ferromagnetic nanoparticles are added.

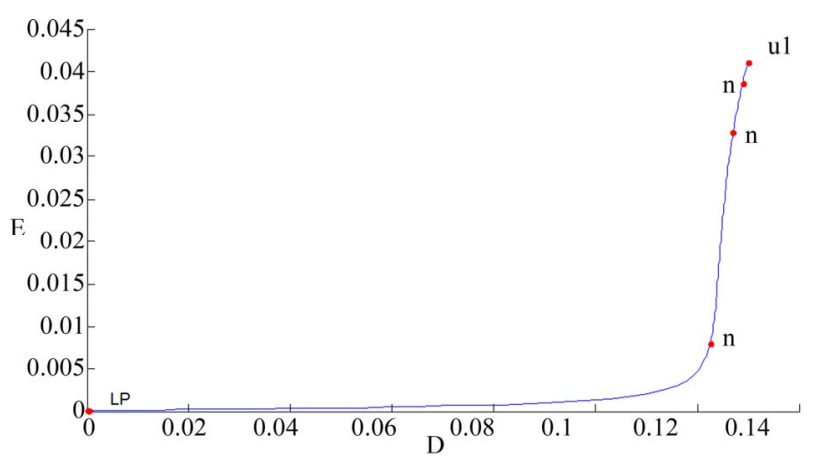

Figure 2. Hopf bifurcation in case 1 dispppears for a nanoparticle mass fraction of $\mathbf{0 . 0 0 6}$.

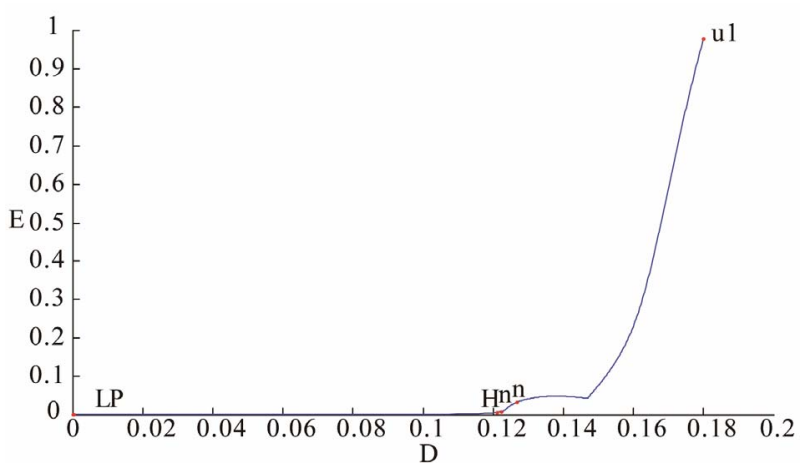

Figure 3. Hopf bifurcation in case 2. 


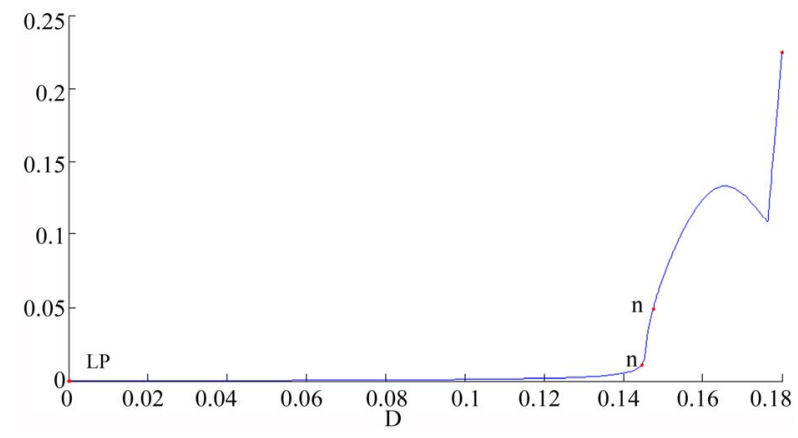

Figure 4. Hopf bifurcation in case 2 eliminated because of nanoparticles $($ mass fraction $=\mathbf{0 . 0 0 5})$.

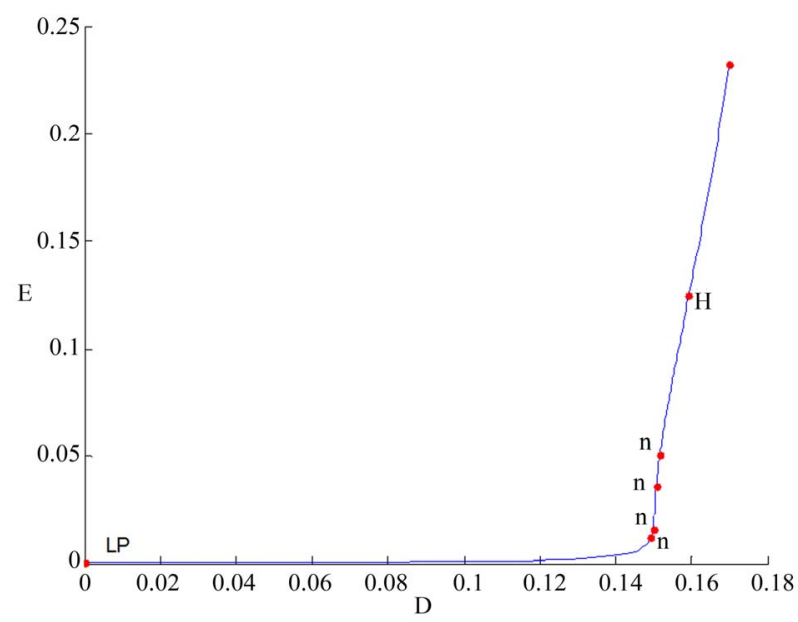

Figure 5. Hopf bifurcation in case 3.

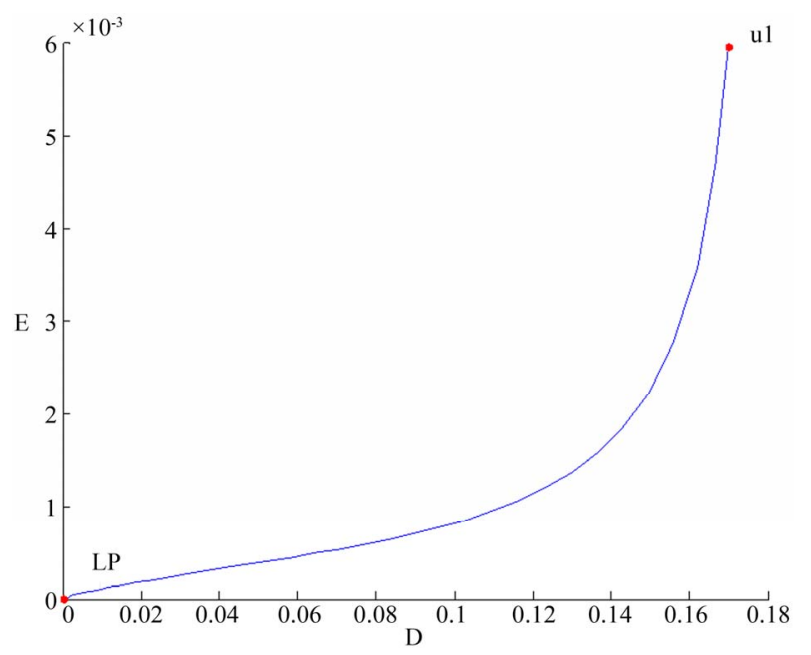

Figure 6. Hopf bifurcation in case 3 eliminated as a result of adding nanoparticles (mass fraction 0.0065 ).

would be more economical than having to continuously supply extra oxygen to eliminate the oscillations [1]. The results discussed in this paper should motivate experimentalists to try to use nanoparticles to eliminate unwanted and wasteful oscillations that occur in fermentation processes.
The Hopf bifurcations that occur in the fermentation processes are extremely sensitive to the change in the mass transfer coefficient. And a small change in the mass transfer coefficient. is enough to remove the Hopf bifurcations and that is the main focus of this paper. Reference [1] actually demonstrated computationally that increasing oxygen would remove the oscillation causing Hopf bifurcations. Oxygen can be increased directly or by increasing the mass transfer coefficient. Nanoparticles definitely increase the mass transfer (either by increasing $k_{L}$ or by increasing the area and therefore they have the ability to remove the oscillations that plague the fermentation process and that is the main focus of this paper. While more advanced and sophisticated co-relations connecting the mass transfer coefficient to the amount of nanoparticles can indeed be developed, the facts are 1) Hopf bifurcations can indeed disappear as a result of the increase of oxygen mass transfer and 2) the oxygen mass transfer coefficient can be enhanced by using a minute amount of nanoparticles. The computational demonstration of these facts is important to guide the experimentalists to perform the necessary experiments to validate the fact that a small amount of nanoparticles can remove the oscillation causing Hopf bifurcations.

\section{Conclusion}

The effect of using ferromagnetic nanoparticles on the oxygen mass transfer coefficient in the cybernetic model for the Saccharomyces cerevisiae fermentation process has been studied. In particular, it is shown that using a minute amount of the ferromagnetic nanoparticles would be effective in the elimination of the unwanted oscillatory behavior in the fermentation process.

\section{REFERENCES}

[1] L. N. Sridhar, "Elimination of Oscillations in Fermentation Processes,” AICHE Journal, Vol. 57, No. 9, 2011, pp. 2397-2405.

[2] B. Olle, S. Bucak, T. C. Holmes, L. Bromberg, A. Hatton and D. I. C. Wang, "Enhancement of Oxygen Mass Transfer Using Functionalized Magnetic Nanoparticles," Industrial \& Engineering Chemistry Research, Vol. 45, No. 12, 2006, pp. 4355-4363.

[3] B. Olle, "Mechanistic Modeling of Increased Oxygen Transport Using Functionalized Magnetic Fluids in Bioreactors," PhD Thesis, Massachusetts Institute of Technology, Cambridge, 2006.

[4] K. D. Jones and D. S. Kompala, "Cybernetic Model of the Growth Dynamics of the Saccharomyces cerevisiae in Batch and Continuous Cultures," Journal of Biotechnology, Vol. 71, No. 1-3, 1999, pp. 105-131. doi:10.1016/S0168-1656(99)00017-6

[5] K. J. Lee, D. E. Tribe and P. L. Rogers, "Ethanol Production by Zymomonas mobilis in Continuous Culture at High 
Glucose Concentrations,” Biotechnology Letters, Vol. 1, No. 10, 1979, pp. 421-426.

[6] K. J. Lee, M. L. Skotnicki, D. E. Tribe and P. L. Rogers, "Kinetic Studies on a Highly Productive Strain of Zymomonas mobilis,” Biotechnology Letters, Vol. 2, No. 8, 1980, pp. 339-344. doi:10.1007/BF00138666

[7] I. M. L. Jobses, G. T. C. Egbertsa, K. C. A. M. Luyben and J. A. Roels, "Fermentation Kinetics of Zymomonas mobilis at High Ethanol Concentrations; Oscillations in Continuous Cultures," Biotechnology and Bioengineering, Vol. 28, No. 6, 1986, pp. 868-877.

[8] C. Ghommidh, J. Vaija, S. Bolarinwa and J. M. Navarro, "Oscillatory Behavior of Zymomonas mobilis in Continuous Cultures: A Simple Stochastic Model,” Biotechnology Letters, Vol. 11, No. 9, 1989, pp. 659-664.

[9] L. J. Bruce, D. B. Axford, B. Ciszek and J. A. Daugulis, "Extractive Fermentation by Zymomonas mobilis and the Control of Oscillatory Behavior," Biotechnology Letters, Vol. 13, No. 4, 1991, pp. 291-296. doi:10.1007/BF01041487

[10] L. Perego, J. M. Cabral, S. Dias, L. H. Koshimizu, M. R. De Melo Cruz, W. Borzani and M. L. R. Vairo, "Influence of Temperature, Dilution Rate and Sugar Concentration on the Establishment of Steady-State in Continuous Ethanol Fermentation of Molasses," Biomass, Vol. 6, No. 3, 1985, pp. 247-256. doi:10.1016/0144-4565(85)90044-7

[11] A. Mulchandani and B. Volesky, "Modelling of the Acetone-Butanol Fermentation with Cell Retention," Canadian Journal of Chemical Engineering, Vol. 64, No. 4, 1986, pp. 625-631.

[12] C. Strassle, B. Sonnleitner and A. A. Fiechter, "A Predic-

\section{Nomenclature}

C: Intracellular Carbohydrate Mass Fraction Storage;

$D$ : Dilution Rate;

$V$ : Volume;

E: Ethanol Concentration; tive Model for the Spontaneous Synchronization of Saccharomyces cerevisiae Grown in Continuous Culture, II. Experimental Verification,” Journal of Biotechnology, Vol. 9, No. 3, 1989, pp. 191-208. doi:10.1016/0168-1656(89)90108-9

[13] H. K. Von Meyenberg, "Stable Synchrony Oscillations in Continuous Culture of Saccharomyces cerevisiae under Glucose Limitation,” In: B. Chance, E. K. Pye, A. K. Shosh and B. Hess, Eds., Biological and Biochemical Oscillators, Academic Press, New York, 1973, pp. 411-417.

[14] Y. Zhang and M. A. Henson, "Bifurcation Analysis of Continuous Biochemical Reactor Models,” Biotechnology Progress, Vol. 17, No. 4, 2001, pp. 647-660. doi:10.1021/bp010048w

[15] D. J. W. Simpson, D. S. Kompala and J. D. Meiss, "Discontinuity Induced Bifurcations in a Model of Saccharomyces cerevisiae," Mathematical Biosciences, Vol. 218, No. 1, 2009, pp. 40-49. doi:10.1016/j.mbs.2008.12.005

[16] A. Dhooge, W. Govearts and A. Y. Kuznetsov, "MATCONT: A Matlab Package for Numerical Bifurcation Analysis of ODEs," ACM Transactions on Mathematical Software, Vol. 29, No. 2, 2003, pp. 141-164. doi:10.1145/779359.779362

[17] A. Dhooge, W. Govaerts, Y. A. Kuznetsov, W. Mestrom, A. M. Riet and CL_MATCONT, "A Continuation Toolbox in Matlab,” 2004.

[18] S. J. Parulekar, G. B. Semones, M. J. Rolf, J. C. Lievense and H. C. Lim, "Introduction and Elimination of Oscillations in Continuous Cultures of Saccharomyces cerevisiae”, Biotechnology and Bioengineering, Vol. 28, No. 5, 1986, pp. 700-710. doi:10.1002/bit.260280509

$X$ : Cell Mass Concentration;

$O$ : Oxygen Concentration;

$O^{*}$ : Oxygen Solubility Limit (7.5);

$Y_{i}: i^{\text {th }}$ Pathway Yield Coefficient. 